\title{
Asymptotic Critical Transmission Radii for Greedy Forward Routing in Wireless Ad Hoc Networks
}

\author{
Peng-Jun Wan, Chih-Wei Yi, Member, IEEE, Lixin Wang, Frances Yao, and Xiaohua Jia
}

\begin{abstract}
In wireless ad hoc networks, greedy forward routing is a localized geographic routing algorithm in which one node discards a packet if none of its neighbors is closer to the destination of the packet than itself, or otherwise forwards the packet to the neighbor closest to the destination. If all nodes have the same transmission radii, the critical transmission radius for greedy forward routing is the smallest transmission radius which ensures packets can be delivered by greedy forward routing through any source-destination pair. In this paper, we study asymptotic critical transmission radii of randomly deployed wireless ad hoc networks. Assume network nodes are represented by a Poisson point process of density $n$ over a unit-area convex compact region whose boundary curvature is bounded. We show that the ratio of critical transmission radii to $\sqrt{\frac{\ln n}{\pi n}}$ is asymptotically almost surely equal to $\sqrt{1 /\left(\frac{2}{3}-\frac{\sqrt{3}}{2 \pi}\right)} \approx 1.6$.
\end{abstract}

Index Terms-Wireless ad hoc networks, greedy forward routing, critical transmission radii, random deployment.

\section{INTRODUCTION}

A wireless ad hoc network is a collection of wireless devices distributed over a geographic region. Each ad hoc device is equipped with an omnidirectional antenna. A communication session is established either through a singlehop radio transmission if the communication party is close enough, or through relaying by intermediate devices otherwise. The selection of intermediate relay nodes is determined by routing algorithms. Greedy forward routing (abbreviated by GFR) is one of the localized geographic routing algorithms proposed in literature.

In GFR, one node discards a packet if none of its neighbors is closer to the destination of the packet than itself, or otherwise forwards the packet to the neighbor closest to the destination. Therefore, each packet should contain the location of its destination, and each node only needs to maintain the locations of its one-hop neighbors. GFR can be implemented in a localized and memoryless manner. There are some variations of GFR. For example, in [1] and [2], the

Paper approved by R. Fantacci, the Editor for Wireless Networks and Systems of the IEEE Communications Society. Manuscript received July 24 , 2006; revised March 18, 2007, December 17, 2007, June 14, 2008, and June 21, 2008.

P.-J. Wan and L. Wang are with the Department of Computer Science, Illinois Institute of Technology, Chicago, IL 60616 (e-mail: wan@cs.iit.edu, wanglix@ iit.edu).

C-W. Yi is with the Department of Computer Science, National Chiao Tung University, Hsinchu City 30010, Taiwan (e-mail: yi@cs.nctu.edu.tw).

F. Yao and X. Jia are with the Department of Computer Science, City University of Hong Kong, Hong Kong (e-mail: \{csfyao, csjia\}@ cityu.edu.hk).

A short version of this paper had been presented in the Seventh ACM International Symposium on Mobile Ad Hoc Networking and Computing (MobiHoc 2006), Florence, Italy, 22-25 May 2006.

Digital Object Identifier 10.1109/TCOMM.2009.05.070307

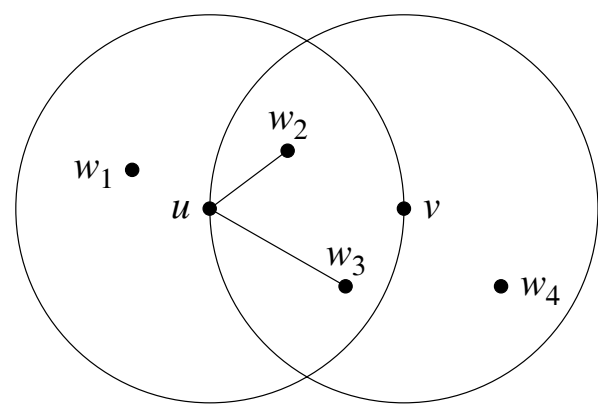

Fig. 1. $\quad u$ is a source node and $v$ is the corresponding destination node.

shortest projected distance to the destination on the straight line joining the current node and the destination node is considered as the greedy metrics. In [1], packets are allowed to be sent backward if there is no forwarding neighbor. In [2], only nodes whose Voronoi cells intersect with the sourcedestination line segment are eligible for being relay nodes. Here the Voronoi cell of a node is the set of points in the plane that are closer to the node than to any other node [3].

Due to existence of local minima where none of neighbors is closer to the destination than the current node, a packet may be discarded before arriving its destination. To ensure that every packet can arrive its destination, all nodes should have sufficiently large transmission radii to avoid being local minima. For points $x, y \in \mathbb{R}^{2}$ and a positive real number $r$, let $B(x, r)$ denote the open disk of radius $r$ centered at $x$, $\|x\|$ denote the Euclidean norm of $x$, and $\|x-y\|$ denote the Euclidean distance between $x$ and $y$. Consider Fig. 1. Let $u$ be a source or relay node, $v$ be the corresponding destination node, and $w_{i}$ denote nodes other than $u$ and $v$. Nodes that can relay packets for $u$ toward $v$ must be in the region $B(u,\|u-v\|) \cap B(v,\|u-v\|)$ based on the following observations. If $w_{i}$ can relay packets for $u$ toward $v$, it must be closer to $v$ than $u$, i.e. $\left\|v-w_{i}\right\|<\|v-u\|$ or equivalently $w_{i} \in B(v,\|u-v\|) . w_{2}, w_{3}, w_{4}$ satisfy this rule and $w_{1}$ does not. On the other hand, if no one can relay packets for $u$, packets should be directly transmitted from $u$ to $v$. So, in the worst case, $u$ at most needs to set its transmission radius to $\|u-v\|$. This implies candidates of relay nodes must be in $B(u,\|u-v\|)$. For example, in Fig. $1, w_{4}$ can't be a candidate of relay nodes. Thus, only $w_{2}$ and $w_{3}$ can relay packets for $u$ toward $v$. In addition, if the transmission radius is set to $\min \left(\left\|w_{2}-u\right\|,\left\|w_{3}-u\right\|\right), u$ has at least one neighbor to relay packets. The procedure of selecting the minimal transmission radii to ensure either $u$ can send packets directly to $v$ or there exists at least one node to relay packets for $u$ 
toward $v$ can be expressed as $\min _{w_{i} \in B(v,\|u-v\|)}\left\|w_{i}-u\right\|$. For a given point set $V$ in the plane, let

$$
\rho(V)=\max _{\substack{(u, v) \in V^{2} \\ u \neq v}}\left(\min _{w \in B(v,\|u-v\|) \cap V}\|w-u\|\right) .
$$

It is the the maximum of $\min _{w \in B(v,\|u-v\|) \cap V}\|w-u\|$ over all $(u, v)$ pairs of nodes.

To eliminate local minima in the network, we choose $\rho(V)$ as the transmission radius. According to the previous discussion, any node $u$ always can deliver packets toward any other node. However, is $\rho(V)$ the optimal (smallest) transmission radius for local-minimum-free? The answer is positive. Consider the pair of nodes $(u, v)$ that gives the value $\rho(V)$. If the transmission radius is set less than $\rho(V), u$ can't directly send packets to $v$ and there is no other node that can relay packets for $u$ toward $v$. So, $u$ is a local minimum w.r.t. $v$. So, $\rho(V)$ is the optimal one and called the critical transmission radius for (local-minimum-free) GFR that guarantees the deliverability of packets. In the rest of this paper, the critical transmission radius for GFR is simply written as the critical transmission radius and abbreviated as CTR.

The analytic work of GFR can be dated back to 1984 by Takagi and Kleinrock [1]. They studied the optimal transmission radius to maximize the expected progress of packets based on most forward and least backward routing strategy in which every node delivers each packet to the neighbor (not including itself) with the shortest projected distance to the destination on the straight line joining the current node. However, the deliverability of packets is not considered. Recently, Xing et al. [2] (2004) show that in a fully covered homogeneous wireless sensor network, if the transmission radius is larger than 2 times of the sensing radius, the deliverability can be guaranteed between any source-destination pair by greedy forwarding schemes in which a packet is sent to the neighbor either with the shortest Euclidean distance to the destination [4] [5] or with the shortest projected distance to the destination on the straight line joining the current node and the destination node [1] and by bounded Voronoi greedy forwarding scheme in which only those nodes whose Voronoi cells intersect with the line segment between the source and destination are eligible to relay the packet.

Another related and interesting problem in literature is the longest edge of connected geometric graphs. Penrose [6] (1997) [7] (1999) studied the longest edge of a minimal spanning tree which is corresponding to the critical transmission radius for connectivity in random geometric graphs. Later, by applying the percolation theory, Gupta and Kumar [8] had similar results for wireless networks. Recently, Baccelli and Bordenave [9] (2007) introduced a structure called radial spanning trees (RSTs) in which each node, excluding the root $s$ at the origin of the plane, has an edge to its closest neighbor among nodes closer to the root $s$. The length of the longest edge of RSTs can be given by $\max _{u \in V, u \neq s} \min _{w \in B(s,\|s-u\|) \cap V}\|w-u\|$. If $s$ is the only destination, then the value is the critical transmission radius for local-minimum-free GFR.

In this paper, we study the deliverability by giving the asymptotics of $\rho(V)$ where $V$ is a Poisson point process.
Assume that the deployment region $\mathbb{D}$ is a convex compact region whose boundary has bounded curvature. By scaling, we assume $\mathbb{D}$ have unit area. Let $\mathcal{P}_{n}$ denote a Poisson point process of density $n$ over $\mathbb{D}$. The ratio of $\rho\left(\mathcal{P}_{n}\right)$ to $\sqrt{\frac{\ln n}{\pi n}}$ is asymptotically almost surely equal to $\sqrt{1 /\left(\frac{2}{3}-\frac{\sqrt{3}}{2 \pi}\right)} \approx 1.6$.

The rest of this paper is organized as follows. In Section II, we present our main results and show some possible applications. In Section III, the proof of main results is given, but most calculation details and related geometric and probabilistic lemmas are left in the appendix. In Section IV, simulation results were given to evidence our asymptotic analysis. Our conclusions are in Section V.

\section{Main Results}

Let $\mathbb{D}$ be a unit-area convex compact region with a boundedcurvature boundary, and $\mathcal{P}_{n}$ denote a Poisson point process of density $n$ over $\mathbb{D}$. Let $\beta_{0}=1 /\left(\frac{2}{3}-\frac{\sqrt{3}}{2 \pi}\right) \approx 1.6^{2}$. The main result of this paper is the following theorem.

Theorem 1: For any $\varepsilon>0$,

$\lim _{n \rightarrow \infty} \operatorname{Pr}\left[(1-\varepsilon) \sqrt{\frac{\beta_{0} \ln n}{n \pi}} \leq \rho\left(\mathcal{P}_{n}\right) \leq(1+\varepsilon) \sqrt{\frac{\beta_{0} \ln n}{n \pi}}\right]=1$.

Since the converge is in probability, we remark Theorem 1 can't be simplified to $\lim _{n \rightarrow \infty} \rho\left(\mathcal{P}_{n}\right)=\sqrt{\frac{\beta_{0} \ln n}{n \pi}}$. Based on Theorem 1, we have the following corollary.

Corollary 2: If the transmission radius is set to $\sqrt{\frac{\beta \ln n}{\pi n}}$ for some constant $\beta$, we have

1) If $\beta>\beta_{0}$, it is asymptotic almost sure that packets can be delivered by GFR between any pair of nodes. ${ }^{1}$

2) If $\beta<\beta_{0}$, it is asymptotic almost sure that packets can't be delivered by GFR between some pairs of nodes.

Possible Applications: In the rest of this section, we show some possible applications of Theorem 1. Due to harsh deployment environment coupled with a large amount of sensors to be deployed, random deployment is unavoidable in many applications of wireless ad hoc and sensor networks. At the same time, owing to the constraint on the maximal transmission power, each wireless device can only communicate with nearby nodes, and therefore connectivity of network topology and deliverability of routing protocols are the most important issue of randomly deployed networks. Our asymptotic research results associated with simulation data can be a good reference to the following problems and help us to improve energy efficiency.

- Maximal transmission power: According to path loss models of wireless communications, the maximal transmission power is strongly related to the maximal transmission radius and is a key parameter during the design phase of wireless devices. The choosing of the maximal transmission power can base on the maximal transmission radius. Our results show that $\Theta\left(\sqrt{\frac{\ln n}{n}}\right)$ is a good reference for choosing the maximal transmission radius. ${ }^{2}$

\footnotetext{
${ }^{1}$ An event is said to be asymptotic almost sure (abbreviated by a.a.s.) if it occurs with a probability converges to one as $n \rightarrow \infty$.

${ }^{2}$ For two sequences $f_{n}$ and $g_{n}$, we write $f_{n}=\Theta\left(g_{n}\right)$ if there exist constants $c_{1}>0, c_{2}$ and $n_{0}$ such that $c_{1}\left|g_{n}\right| \leq\left|f_{n}\right| \leq c_{2}\left|g_{n}\right|$ for all $n \geq n_{0}$.
} 


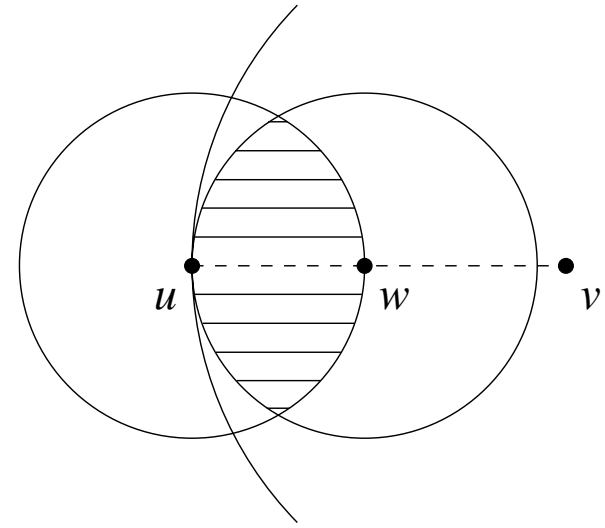

Fig. 2. $w$ is the intersection point of the segment $u v$ and the circle $B\left(u, r_{n}\right)$. The shaded area is $B\left(u, r_{n}\right) \cap B\left(w, r_{n}\right)$ which is contained in $B\left(u, r_{n}\right) \cap$ $B(v,\|u-v\|)$.

- The critical number of nodes: To deploy a WSN over a region, if the transmission range of nodes is known, we need to decide how many sensor nodes are enough such that the network can be connected by routing algorithms. By scaling the deployment region to unit-area and also scaling the transmission radius by the same ratio, we can have a critical number of nodes based on the theoretical formula or simulation data.

- Light-weight routing algorithms: If geographic information is available, greedy forward routing is easy to implement and requires few resources, but suffers from local minimum problems. Therefore, some relatively complex compensatory algorithms are needed to handle such exceptional situations. If the delivery rate can be predicted and controlled above tolerable level or even more the deliverability can be guaranteed, the pure greedy forward routing is enough, and complex compensatory algorithms are not necessary.

\section{Outline OF Proof}

This section is dedicated to the proof of Theorem 1 .

\section{A. Upper Bounds for the Critical Transmission Radius}

For a given $\varepsilon>0$, let $\beta=(1+\varepsilon)^{2} \beta_{0}$. The upper bound for $\rho\left(\mathcal{P}_{n}\right)$ given in Theorem 1, i.e. $\rho\left(\mathcal{P}_{n}\right) \leq$ $(1+\varepsilon) \sqrt{\frac{\beta_{0} \ln n}{n \pi}}$, can be proved by showing that if $r_{n}=$ $\sqrt{\frac{\beta \ln n}{\pi n}}=(1+\varepsilon) \sqrt{\frac{\beta_{0} \ln n}{n \pi}}$, there a.a.s. don't exist local minima. For a pair of nodes $(u, v), u$ is a local minimum w.r.t. $v$ if and only if $\|u-v\|>r_{n}$ and there are no other nodes in $B\left(u, r_{n}\right) \cap B(v,\|u-v\|)$. Now, assume $\|u-v\|>r_{n}$ and let $w$ be the intersection point of the segment $u v$ and the circle $\partial B\left(u, r_{n}\right)$. See Fig. 2. For convenience, for any two points $x, y \in R^{2}$, the region $B(x,\|x-y\|) \cap B(y,\|x-y\|)$, denoted by $L_{x y}$, is called the lune associated with $x$ and $y$, and the segment $x y$ is called the waist of $L_{x y}$. Since $L_{u w} \subset B\left(u, r_{n}\right) \cap B(v,\|u-v\|)$, "there exist nodes in $L_{u w} "$ implies $" u$ is not a local minimum w.r.t. $v$ ". We shall show that any lune whose waist is of length $r_{n}$, e.g. like $L_{u w}$, a.a.s. covers some nodes. Thus, the network is local-minimum-free.

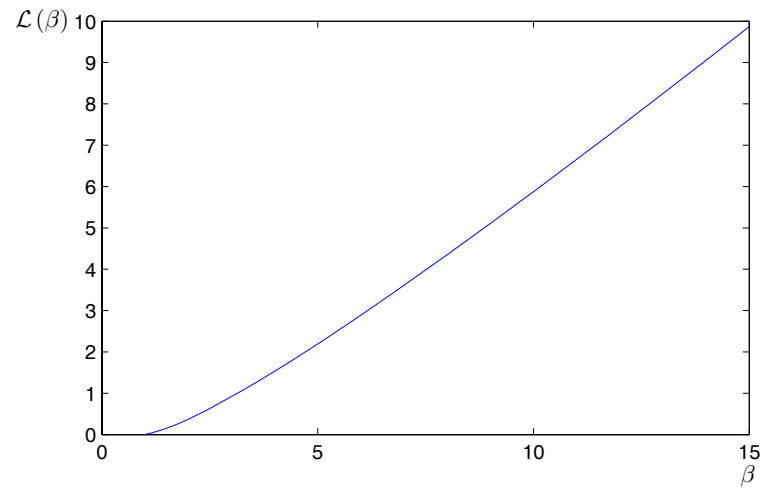

Fig. 3. The graph of $\mathcal{L}(\beta)$.

We use \# $(S)$ to denote the cardinality of a countable set $S$. For any finite point set $V \subset \mathbb{D}$ and any $r>0$, define

$$
\mathcal{S}(V, r)=\min _{u, v \in \mathbb{D},\|u-v\|=r} \#\left(V \cap L_{u v}\right) .
$$

$\mathcal{S}(V, r)$, called the minimal scan statistics, is the minimal number of nodes of $V$ that can be covered by a lune whose waist is fully contained in $\mathbb{D}$ and with length $r$. So, the event $\mathcal{S}\left(\mathcal{P}_{n}, r_{n}\right)>0$ implies the event $\rho\left(\mathcal{P}_{n}\right) \leq r_{n}$. An a.a.s. lower bound for $\mathcal{S}\left(\mathcal{P}_{n}, r_{n}\right)$ will be given in Lemma 3 and implies that if $\beta>\beta_{0}, \mathcal{S}\left(\mathcal{P}_{n}, r_{n}\right)>0$ is a.a.s..

Let $\phi(\mu)$ denote the function $\phi(\mu)=1-\mu+\mu \ln \mu$ over $\mu \in(0, \infty)$. $\phi$ is strictly convex and has the unique minimum zero at $\mu=1$. Let $\phi^{-1}:[0,1) \rightarrow(0,1]$ be the inverse of the restriction of $\phi$ to $(0,1]$. We define a function $\mathcal{L}$ over $(0, \infty)$ by

$$
\mathcal{L}(\beta)= \begin{cases}\beta \phi^{-1}(1 / \beta) & \text { if } \beta>1, \\ 0 & \text { otherwise. }\end{cases}
$$

The graph of $\mathcal{L}(\beta)$ is illustrated in Fig. 3. We have the following lemma.

Lemma 3: Suppose that $n \pi r_{n}^{2}=(\beta+o(1)) \ln n$ for some $\beta>\beta_{0} .{ }^{3}$ Then for any constant $\beta_{1} \in\left(\beta_{0}, \beta\right)$, it is a.a.s. that

$$
\mathcal{S}\left(\mathcal{P}_{n}, r_{n}\right)>\frac{1}{2} \mathcal{L}\left(\frac{\beta_{1}}{\beta_{0}}\right) \ln n>0 .
$$

A proof of Lemma 3 is given in the appendix and also can be found in [10]. According to Lemma 3, we have $\rho\left(\mathcal{P}_{n}\right) \leq$ $r_{n}=(1+\varepsilon) \sqrt{\frac{\beta_{0} \ln n}{\pi n}}$ is a.a.s..

\section{B. Lower Bounds for the Critical Transmission Radius}

The lower bound for $\rho\left(\mathcal{P}_{n}\right)$ given in Theorem 1, i.e. $(1-\varepsilon) \sqrt{\frac{\beta_{0} \ln n}{n \pi}} \leq \rho\left(\mathcal{P}_{n}\right)$, will be proved in this subsection. For a given $\varepsilon>0$, let $\beta=(1-\varepsilon)^{2} \beta_{0}$. The lower bound can be proved by showing that if $r_{n}=\sqrt{\frac{\beta \ln n}{\pi n}}=$ $(1-\varepsilon) \sqrt{\frac{\beta_{0} \ln n}{n \pi}}$, there a.a.s. exist local minima. The plane is going to be tessellated into equal-size square cells. For each cell, an event that implies existence of local minima within the cell is introduced, and a lower bound for the probability of the event is derived. Since these events are identical and

\footnotetext{
${ }^{3}$ For two sequences $f_{n}$ and $g_{n}$, we write $f_{n}=o\left(g_{n}\right)$ if $\lim _{n \rightarrow \infty} \frac{f_{n}}{g_{n}}=0$.
} 
independent among cells, we can estimate an low bound for the probability of existence of local minima in the network, and prove the lower bound is a.a.s. equal to 1 .

Let $\beta_{1}$ and $\beta_{2}$ be two positive constants such that

$$
\begin{gathered}
\max \left(\frac{1}{4} \beta_{0}, \beta\right)<\beta_{1}<\beta_{2}<\beta_{0}, \text { and } \\
\frac{\pi^{2}}{c^{2}}\left(1-\frac{\sqrt{\beta_{1}}}{\sqrt{\beta_{2}}}\right)<1 .
\end{gathered}
$$

Here $c$ is the constant in Lemma 6 that is given in Appendix. Let $R_{1}(n)$ and $R_{2}(n)$ be given by

$$
n \pi\left(R_{1}(n)\right)^{2}=\beta_{1} \ln n \text { and } n \pi\left(R_{2}(n)\right)^{2}=\beta_{2} \ln n .
$$

Divide $\mathbb{D}$ by a $\left(4 \sqrt{\frac{\ln n}{n \pi}}\right)$-tessellation. ${ }^{4}$ Let $I_{n}$ denote the number of cells fully contained in $\mathbb{D}$, and we have

$$
I_{n}=\Theta\left(\frac{n}{\ln n}\right) .
$$

For each cell fully contained in $\mathbb{D}$, we draw a disk with radius $\frac{1}{2} \sqrt{\frac{\ln n}{n \pi}}$ at the center of the cell. For $1 \leq i \leq I_{n}$, let $E_{i}$ be the event that there exist two nodes $X, Y \in \mathcal{P}_{n}$ such that their midpoint is in the $i$-th disk and their distance is between $R_{1}(n)$ and $R_{2}(n)$, and there is no other node in the lune $L_{X Y}$. For any two nodes $u$ and $v$ with $\|u-v\|>r_{n}$, if there is no other node in $L_{u v}, u$ and $v$ are local minima w.r.t. each other. So, $E_{i}$ implies existence of local minima and

$$
\operatorname{Pr}\left[\rho\left(\mathcal{P}_{n}\right)>r_{n}\right] \geq \operatorname{Pr}\left[\text { at least one } E_{i} \text { occurs] } .\right.
$$

Let $o_{i}$ denote the center of the $i$-th disk, and $u, v$ be two points such that their midpoint is on the disk and their distance is between $R_{1}(n)$ and $R_{2}(n)$. (See Fig. 4.) Since the middle point of $u$ and $v$, called $z$, is in the disk, we have $\left\|o_{i}-z\right\| \leq$ $\frac{1}{2} \sqrt{\frac{\ln n}{n \pi}}$. For any point $w \in L_{u v}$, the distance between $w$ and $z$, i.e. $\|w-z\|$, is at most $\frac{\sqrt{3}}{2}\|u-v\| \leq \frac{\sqrt{3}}{2} \sqrt{\frac{\beta_{0} \ln n}{n \pi}}$. For any point $w \in L_{u v}$, applying triangle inequality, we have

$$
\begin{aligned}
\left\|w-o_{i}\right\| & \leq\|w-z\|+\left\|o_{i}-z\right\|<\frac{\sqrt{3 \beta_{0}}}{2} \sqrt{\frac{\ln n}{n \pi}}+\frac{1}{2} \sqrt{\frac{\ln n}{n \pi}} \\
& \approx 1.885 \sqrt{\frac{\ln n}{n \pi}}<2 \sqrt{\frac{\ln n}{n \pi}} .
\end{aligned}
$$

Since the cell width is $4 \sqrt{\frac{\ln n}{n \pi}}, u, v$ and $L_{u v}$ are contained in the $i$-th cell. Therefore, $E_{1}, \cdots, E_{I_{n}}$ are independent. In addition, $E_{1}, \cdots, E_{I_{n}}$ are identical. Then,

$$
\left.\operatorname{Pr} \text { [none of } E_{i} \text { occurs }\right]=\left(1-\operatorname{Pr}\left[E_{1}\right]\right)^{I_{n}} \leq e^{-I_{n} \operatorname{Pr}\left(E_{1}\right)} .
$$

If $I_{n} \operatorname{Pr}\left(E_{1}\right) \rightarrow \infty$, then $\operatorname{Pr}\left[\rho\left(\mathcal{P}_{n}\right)>r_{n}\right] \rightarrow 1$ follows, and from Eq. (5), the lower bound for $\rho\left(\mathcal{P}_{n}\right)$ in Theorem 1 is obtained. So, we only need to prove the following lemma.

Lemma 4: $I_{n} \operatorname{Pr}\left(E_{1}\right) \rightarrow \infty$.

The proof of Lemma 4 is given in the appendix and also can be found in [10].

\footnotetext{
${ }^{4} \mathrm{An} \varepsilon$-tessellation is a technique that divides the plane by vertical and horizontal lines into a grid in which each grid cell has width $\varepsilon$.
}

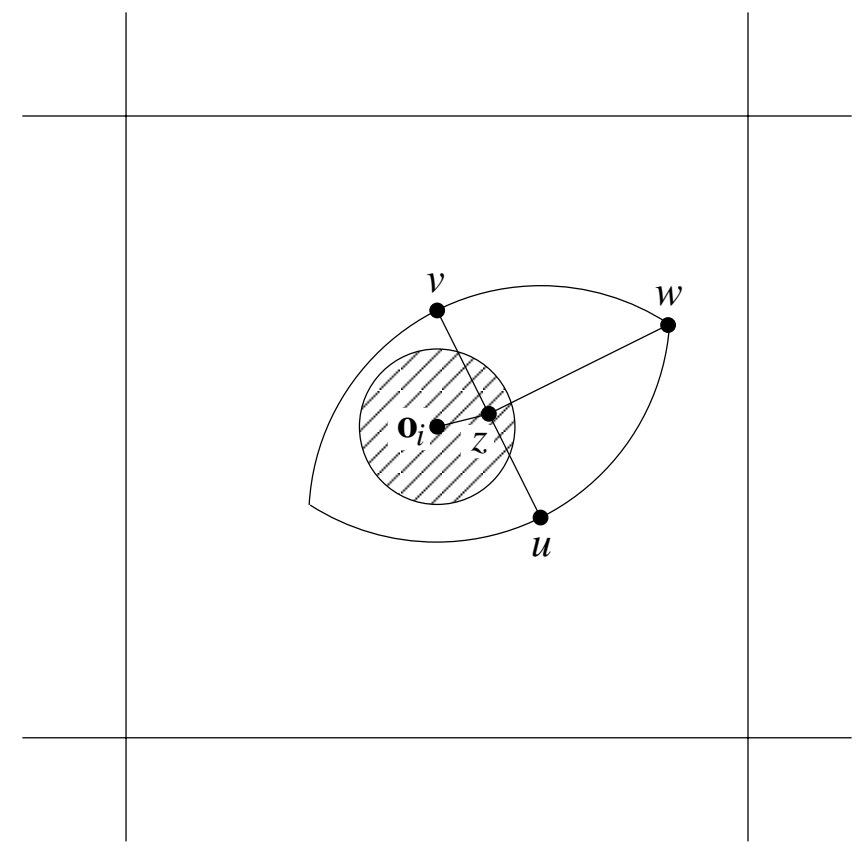

Fig. 4. The cell width is $4 \sqrt{\frac{\ln n}{n \pi}}, o_{i}$ is the center of the cell, and $R_{1}(n)<$ $\|u-v\|<R_{2}(n)$. The disk is centered at $o_{i}$ and with radius $\frac{1}{2} \sqrt{\frac{\ln n}{n \pi}}$, and $z$ is the middle point of $u$ and $v . L_{u v}$ is fully contained in the cell.

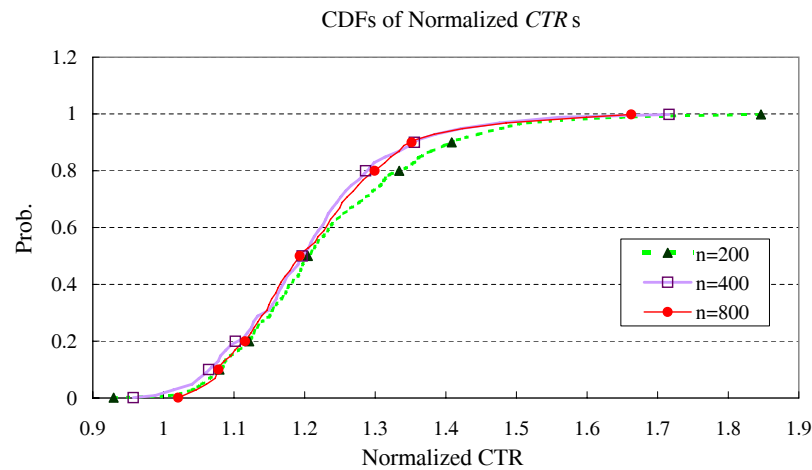

Fig. 5. The cumulative distributed functions of normalized $C T R$ s for $n=$ 200,400 , and 800 .

\section{Simulations}

In the simulation, networks are composed of 200,400 , or 800 nodes distributed over a unit-area disk. Let $n$ denote the network size, i.e. the number of nodes in a network. For each network size, 400 topologies are generated by uniform random point processes. For each network topology, the actual critical transmission radius, denoted by $C T R$, is computed according to Eq. (1). To avoid ambiguity, the estimated (or theoretical) critical transmission radius given by Theorem 1 is denoted by $\rho_{n}$.

First, we would like to observe the trend of convergence of $C T R$ s. For $n=200,400$, and 800 respectively, the average $C T R \mathrm{~s}$ are $0.1808,0.1332$, and 0.1000 , and the theoretical radius $\rho_{n}$ are $0.1469,0.1104$, and 0.0825 . To have a fair comparison over different network sizes, $C T R \mathrm{~s}$ are normalized by being divided by the corresponding $\rho_{n}$. The CDFs of normalized $C T R$ s are illustrated in Fig. 5. The 


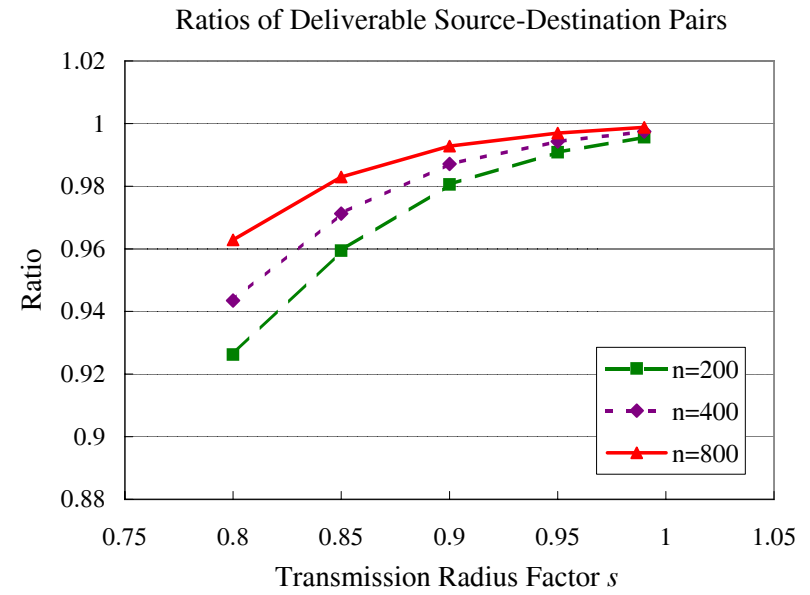

Fig. 6. Average percentage of deliverable source-destination pairs in networks with $n=200, n=400$, and $n=800$.

bold green dotted line marked by triangles is the CDF of normalized $C T R$ s for $n=200$, the bold solid purple line marked by squares is for $n=400$, and the fine solid red line marked by circles is for $n=800$. For each network size, the transition width is the difference between the largest and smallest CTRs among 400 network topologies. The normalized transition width for $n=200$ (respectively, 400 and 800 ) is 0.9168 (respectively, 0.7591 and 0.6419 ) that is the horizontal distance between the right most and left most triangle (respectively, square and circle) markers in Fig. 5. The decreasing of the normalized transition width agrees with the trend of convergence.

Next, if transmission radii are set below $C T R \mathrm{~s}$, we would like to investigate the impact on the deliverability of GFR. Since $C T R$ s usually are different from one topology to another, to have a comparison basis, for each network topology, the $C T R$ is first computed according to Eq. (1), and then transmission radii are set to $s$ times of the $C T R$ for $s=0.8,0.85,0.9,0.95$, or 0.99 . In other words, for each network topology, according to its $C T R$, transmission radii are set to $0.8 \cdot C T R, 0.85 \cdot C T R, 0.9 \cdot C T R, 0.95 \cdot C T R$, or $0.99 \cdot C T R$. The number of deliverable source-destination pairs in each network is counted. For each transmission radius factor $s$, the average ratio of deliverable source-destination pairs are calculated over 400 network topologies. In Fig. 6, the $x$-axis represents the transmission radius factor $s$, and the $y$-axis is the average ratio of deliverable source-destination pairs. We can see that transmission radii have larger impact on deliverability in sparse networks than in dense ones.

Last, we investigate the delivery efficiency of GFR. The effective progress ratio (EPR) of a routing path is defined as the ratio of the Euclidean source-destination distance to the total Euclidean path length. The ratio can be an indicator of delivery efficiency. In the simulation, we calculated average EPRs under various transmission radii and node densities. Similarly, for each network topology, the $C T R$ was first calculated, and then transmission radii are set to $s$ times of the $C T R$. Here $s$ are $0.8,0.9,1,1.1,1.2$, and 1.3. In Fig 7, the $x$ axis represents the transmission radius factor $s$, and the $y$-axis

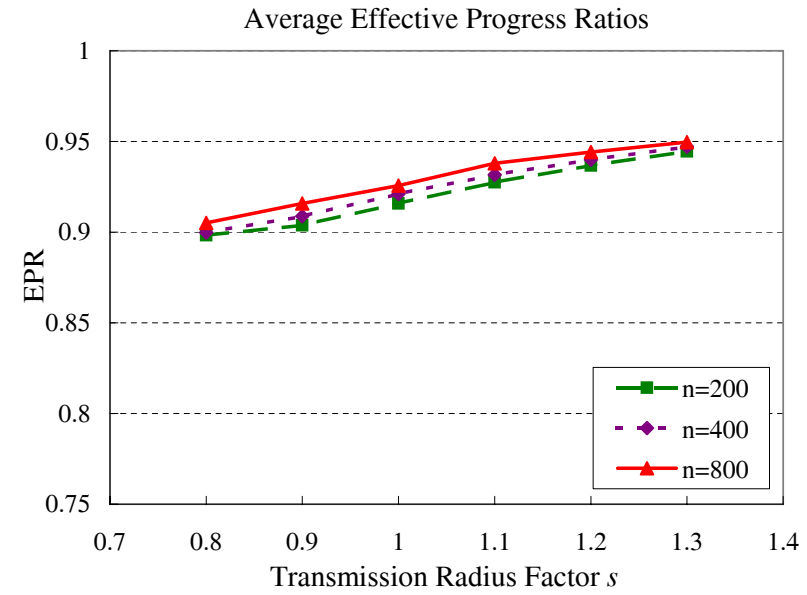

Fig. 7. Effective progress ratios (EPRs) under various transmission radii and network sizes.

is the average EPR over all deliverable source-destination pairs in 400 network topologies. We can see that the EPR mainly depends on the transmission radius factor $s$ but is insensitive to the network size. If the EPR is a major concern, transmission radii will be one of the primary parameters to tune the system.

\section{CONCLUSiOnS}

Greedy forward routing is a localized and memoryless geographic routing algorithm. However, it cannot guarantee the deliverability of packets if transmission radii of nodes are not large enough. If all nodes have the same transmission radii, the smallest transmission radius that ensures the deliverability of packets is referred to as the critical transmission radius. In this paper, we provides tight a.a.s. bounds for the critical transmission radius of randomly deployed wireless ad hoc networks in which nodes are represented by a Poisson point process. We also investigated a number of parameters related to GFR by simulations, including the average of onehop progress, the expected number of hops between sourcedestination pairs, and the effective hop progress. As a future work, it is interesting to study the asymptotics of other localized geographic routing protocols.

\section{APPENDIX}

In the appendix, we give the proof of Lemma 3 and 4. In what follows, $|A|$ is shorthand for 2-dimensional Lebesgue measure (or area) of a measurable set $A \subset \mathbb{R}^{2}$. All integrals considered will be Lebesgue integrals. The diameter of a set $A \subset \mathbb{R}^{2}$ is denoted by diam $(A)$. The topological boundary of a set $A \subset \mathbb{R}^{2}$ is denoted by $\partial A$. $\operatorname{Po}(\lambda)$ represents a Poisson RV with mean $\lambda$. The symbols $O, \Theta, \Omega, o, \sim$ always refer to the limit $n \rightarrow \infty$. To avoid trivialities, we tacitly assume $n$ to be sufficiently large if necessary. For simplicity of notation, the dependence of sets and random variables on $n$ will be frequently suppressed.

\section{A. Geometric Preliminaries}

The lemmas given in this subsection are from [10], and we will skip their proof. If $\|u-v\|=1 / \sqrt{\pi}$, a straightforward 


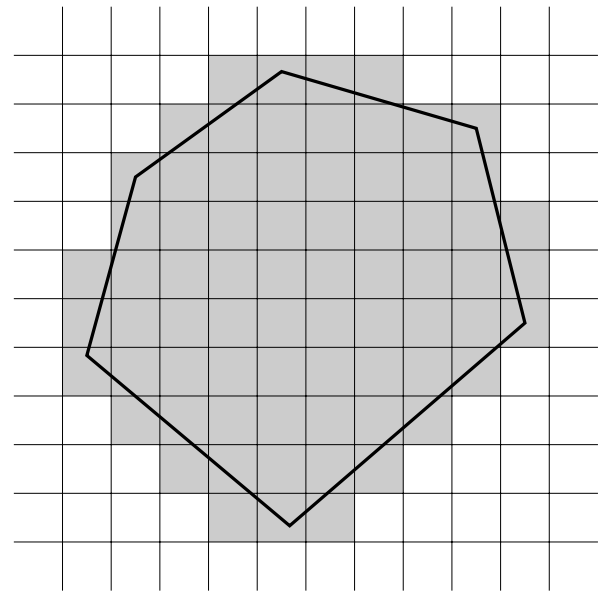

Fig. 8. The cells intersecting with the polygon form a polyquadrate.

calculation yields that $\left|L_{u v}\right|=\frac{2}{3}-\frac{\sqrt{3}}{2 \pi}=\frac{1}{\beta_{0}}$. Let $R_{0}$ denote the minimum of the radius of curvature over $\partial \mathbb{D}$. We have the following lemma.

Lemma 5: For any $u, v \in \mathbb{D}$, if $\|u-v\| \leq R_{0}$ then

$$
\left|L_{u v} \cap \mathbb{D}\right| \geq\left|L_{u v}\right| / 2 .
$$

For two nearby lunes, we use the following lemma to estimation their areas.

Lemma 6: Assume $c=0.039, R>0$, and $a_{1}, b_{1}, a_{2}, b_{2} \in$ $\mathbb{R}^{2}$. Let $z_{1}=\frac{1}{2}\left(a_{1}+b_{1}\right), r_{1}=\left\|a_{1}-b_{1}\right\|, z_{2}=\frac{1}{2}\left(a_{2}+b_{2}\right)$, and $r_{2}=\left\|a_{2}-b_{2}\right\|$. If $r_{1}, r_{2} \in\left[\frac{1}{2} R, R\right],\left\|z_{1}-z_{2}\right\| \leq \sqrt{3} R$, $a_{1}, b_{1} \notin L_{a_{2} b_{2}}$, and $a_{2}, b_{2} \notin L_{a_{1} b_{1}}$, then

$$
\left|L_{a_{1} b_{1}} \cup L_{a_{2} b_{2}}\right|-\left|L_{a_{1} b_{1}}\right| \geq c R\left\|z_{1}-z_{2}\right\| \text {. }
$$

For any convex compact set $C \subset \mathbb{R}^{2}$, we use $C_{-r}$ to denote the set of points of $C$ that are away from $\partial C$ by at least $r$.

Lemma 7: Suppose that $C \subset \mathbb{R}^{2}$ is a convex compact set with diameter at most $d$. Then,

$$
\left|C_{-r}\right| \geq|C|-\pi d r .
$$

An $\varepsilon$-tessellation divides the plane by vertical and horizontal lines into a grid in which each grid cell has width $\varepsilon$. Without loss of generality, we assume the origin is a corner of cells. In a tesselation, a polyquadrate is a collection of cells intersecting with a convex compact set. For example, in Fig. 8, the shaded cells form a polyquadrate induced by a polygon. The horizontal span of a polyquadrate is the horizontal distance measured in the number of cells from the left to the right. The vertical span of a polyquadrate is defined similarly but in the vertical direction. If the span of a convex compact set is $s$ and the width of each cell is $l$, the span of the corresponding polyquadrate is at most $\lceil s / l\rceil+1$.

Lemma 8: If $S$ consists of $m$ cells and $\tau$ is a positive integer constant, the number of polyquadrates with span at most $\tau$ and intersecting with $S$ is $\Theta(m)$.

Now, we introduce a technique to obtain the Jacobian determinant in the change of variables that will be implicitly used in the proof of Lemma 4. Assume a tree topology is fixed over $x_{1}, x_{2}, \cdots, x_{k} \in \mathbb{R}^{2}$. Without loss of generality, we may assume $\left(x_{k-1}, x_{k}\right)$ is one of edges. Let $z_{k-1}=$ $\frac{1}{2}\left(x_{k-1}+x_{k}\right), r=\frac{1}{2}\left\|x_{k}-x_{k-1}\right\|$, and $\theta$ be the slope of $x_{k-1} x_{k}$. For $1 \leq i \leq k-2$, we use $p\left(x_{i}\right)$ to denote $x_{i}$ 's parent in the tree rooted at $x_{k}$, and let $z_{i}=\frac{1}{2}\left(x_{i}+p\left(x_{i}\right)\right)$. Let $I_{2}$ denote a $2 \times 2$ identity matrix and 0 denote a $2 \times 2$ zero matrix. Then, the Jacobian determinant for changing variables $x_{1}, \cdots, x_{k-1}, x_{k}$ by $z_{1}, \cdots, z_{k-1},(r, \theta)$ is

$$
\begin{aligned}
& \left|\frac{\partial\left(x_{1}, \cdots, x_{k-1}, x_{k}\right)}{\partial\left(z_{1}, \cdots, z_{k-1}, r, \theta\right)}\right| \\
& =\left|\frac{\partial\left(x_{1}+p\left(x_{1}\right), \cdots, x_{k-1}+p\left(x_{k-1}\right), x_{k}\right)}{\partial\left(z_{1}, \cdots, z_{k-1}, r, \theta\right)}\right| \\
& =4^{k-1}\left|\frac{\partial\left(\frac{x_{1}+p\left(x_{1}\right)}{2}, \cdots, \frac{x_{k-1}+p\left(x_{k-1}\right)}{2}, x_{k}\right)}{\partial\left(z_{1}, \cdots, z_{k-1}, r, \theta\right)}\right| \\
& =4^{k-1}\left|\frac{\partial\left(z_{1}, \cdots, z_{k-1}, x_{k}-z_{k-1}\right)}{\partial\left(z_{1}, \cdots, z_{k-1}, r, \theta\right)}\right| \\
& =4^{k-1}\left|\begin{array}{ccccc}
I_{2} & \cdots & \mathbf{0} & \mathbf{0} \\
\vdots & \ddots & \vdots & \vdots \\
\mathbf{0} & \cdots & I_{2} & \mathbf{0} \\
\mathbf{0} & \cdots & \mathbf{0} & \cos \theta & -r \sin \theta \\
\sin \theta & r \cos \theta
\end{array}\right|=4^{k-1} r .
\end{aligned}
$$

In the first equality, each non-root variable is added by its parent variable. The equality stands since the Jacobian determinant is equal to 1 as we add one variable to another. We remark if the function to be integrated is independent of the variable $\theta$, then after changing variables, the integral over $\theta$ is equal to $2 \pi$. Actually, this is the most case in this paper.

\section{B. Preliminaries of Poisson RVs}

We first present an estimation of the lower-tail distribution of Poisson RVs.

Lemma 9: For any $\mu \in(0,1)$,

$$
\lim _{\lambda \rightarrow \infty} \operatorname{Pr}(\operatorname{Po}(\lambda) \leq \mu \lambda)=\frac{1}{\sqrt{2 \pi}} \frac{1}{\sqrt{\mu}(1-\mu)} \frac{1}{\sqrt{\lambda}} e^{-\lambda \phi(\mu)} .
$$

Proof: In this proof, the symbol $\sim$ refers to the limit $\lambda \rightarrow \infty$. First, for any $\mu \in(0,1)$, we show that the lower tail distribution of a Poisson RV can be given by

$$
\operatorname{Pr}(\operatorname{Po}(\lambda) \leq \mu \lambda) \sim \frac{1}{1-\mu} \operatorname{Pr}(\operatorname{Po}(\lambda)=\mu \lambda) .
$$

Since

$$
\frac{\operatorname{Pr}(\operatorname{Po}(\lambda)=k-1)}{\operatorname{Pr}(\operatorname{Po}(\lambda)=k)}=\frac{\frac{\lambda^{k-1}}{(k-1) !} e^{-\lambda}}{\frac{\lambda^{k}}{k !} e^{-\lambda}}=\frac{k}{\lambda},
$$

we have

$$
\begin{aligned}
& \operatorname{Pr}(\operatorname{Po}(\lambda) \leq \mu \lambda)=\sum_{k=\mu \lambda}^{0} \operatorname{Pr}(\operatorname{Po}(\lambda)=k) \\
& =\sum_{k=0}^{\mu \lambda} \frac{k !\left(\begin{array}{c}
\mu \lambda \\
k
\end{array}\right)}{\lambda^{k}} \operatorname{Pr}(\operatorname{Po}(\lambda)=\mu \lambda) \\
& \sim \sum_{k=0}^{\mu \lambda} \frac{(\mu \lambda)^{k}}{\lambda^{k}} \operatorname{Pr}(\operatorname{Po}(\lambda)=\mu \lambda) \sim \frac{1}{1-\mu} \operatorname{Pr}(\operatorname{Po}(\lambda)=\mu \lambda) .
\end{aligned}
$$


By Sterling's formula, we have

$$
\begin{aligned}
\operatorname{Pr}(\operatorname{Po}(\lambda) \leq \mu \lambda) & \sim \frac{1}{1-\mu} \frac{\lambda^{\mu \lambda}}{(\mu \lambda) !} e^{-\lambda} \\
& \sim \frac{1}{1-\mu} \frac{\lambda^{\mu \lambda}}{\sqrt{2 \pi \mu \lambda}(\mu \lambda)^{\mu \lambda} e^{-\mu \lambda}} e^{-\lambda} \\
& =\frac{1}{1-\mu} \frac{1}{\sqrt{2 \pi \mu \lambda} \mu^{\mu \lambda}} e^{-\lambda+\mu \lambda} \\
& =\frac{1}{1-\mu} \frac{1}{\sqrt{2 \pi \mu \lambda}} e^{-\lambda+\mu \lambda-\mu \lambda \ln \mu} \\
& =\frac{1}{\sqrt{2 \pi}} \frac{1}{\sqrt{\mu}(1-\mu)} \frac{1}{\sqrt{\lambda}} e^{-\lambda(1-\mu+\mu \ln \mu)} \\
& =\frac{1}{\sqrt{2 \pi}} \frac{1}{\sqrt{\mu}(1-\mu)} \frac{1}{\sqrt{\lambda}} e^{-\lambda \phi(\mu)} .
\end{aligned}
$$

Thus, the lemma is proved.

Assume $Y$ is a Poisson RV with large mean. If $Y$ generates an output, the outcome should be close to the mean with high probability. But as $Y$ generates more outputs, the outcomes are more diverse, and the minimum over the outcomes become smaller. Corresponding to this simple observation, the following lemma gives an quantitative result about the minimum over a collection of Poisson RVs and it will be used in the proof of Lemma 3.

Lemma 10: Assume that $\lim _{n \rightarrow \infty} \frac{\lambda_{n}}{\ln n}=\beta$ for some $\beta>$ 1. Let $Y_{1}, Y_{2}, \cdots, Y_{I_{n}}$ be $I_{n}$ Poisson RVs with means at least $\lambda_{n}$.

1) If $I_{n}=o(n \sqrt{\ln n})$, then for any $1<\beta^{\prime}<\beta$, $\min _{i=1}^{I_{n}} Y_{i}>\mathcal{L}\left(\beta^{\prime}\right) \ln n$ a.a.s.

2) If $I_{n}=O\left(\sqrt{\frac{n}{\ln n}}\right)$, then for any $1<\beta^{\prime}<\beta$, $\min _{i=1}^{I_{n}} Y_{i}>\frac{1}{2} \mathcal{L}\left(2 \beta^{\prime}\right) \ln n$ a.a.s.. ${ }^{5}$

Proof: We first assume that $Y_{1}, Y_{2}, \cdots, Y_{I_{n}}$ all have means $\lambda_{n}$. Let $Y$ be a Poisson RV with mean $\lambda_{n}$. We claim that for any $\mu>0$,

$$
\operatorname{Pr}\left[\min _{i=1}^{I_{n}} Y_{i} \leq \mu \lambda_{n}\right] \leq I_{n} \operatorname{Pr}\left[Y \leq \mu \lambda_{n}\right] .
$$

To prove that this holds, let $X_{i}$ be the indicator of the event $Y_{i} \leq \mu \lambda_{n}$. Then $X_{i}$ is a Bernoulli RV with probability $\operatorname{Pr}\left[Y \leq \mu \lambda_{n}\right]$. Let $X=X_{1}+\cdots+X_{I_{n}}$. Then, $\min _{i=1}^{I_{n}} Y_{i} \leq$ $\mu \lambda_{n}$ if and only if $X \geq 1$. By Markov's inequality,

$$
\begin{aligned}
\operatorname{Pr}\left[\min _{i=1}^{I_{n}} Y_{i} \leq \mu \lambda_{n}\right] & =\operatorname{Pr}[X \geq 1] \leq E[X]=\sum_{i=1}^{I_{n}} E\left[X_{i}\right] \\
& =I_{n} \operatorname{Pr}\left[Y \leq \mu \lambda_{n}\right] .
\end{aligned}
$$

Now, assume that $I_{n}=o(n \sqrt{\ln n})$. Since $\mathcal{L}\left(\beta^{\prime}\right)<$ $\mathcal{L}(\beta)=\beta \phi^{-1}(1 / \beta)$, we have $\mathcal{L}\left(\beta^{\prime}\right) / \beta<\phi^{-1}(1 / \beta)$. We choose a constant $\mu \in\left(\mathcal{L}\left(\beta^{\prime}\right) / \beta, \phi^{-1}(1 / \beta)\right)$. Then, $\mu \in(0,1), \mu \beta>\mathcal{L}\left(\beta^{\prime}\right)$ and $\beta \phi(\mu)>1$. Thus, for sufficiently large $n, \mu \lambda_{n} \geq \mathcal{L}\left(\beta^{\prime}\right) \ln n$, which implies that

$$
\begin{aligned}
\operatorname{Pr}\left[\min _{i=1}^{I_{n}} Y_{i} \leq \mathcal{L}\left(\beta^{\prime}\right) \ln n\right] & \leq \operatorname{Pr}\left[\min _{i=1}^{I_{n}} Y_{i} \leq \mu \lambda_{n}\right] \\
& \leq I_{n} \operatorname{Pr}\left[Y \leq \mu \lambda_{n}\right] .
\end{aligned}
$$

${ }^{5}$ For two sequences $f_{n}$ and $g_{n}$, we write $f_{n}=O\left(g_{n}\right)$ if there exist constants $c$ and $n_{0}$ such that $\left|f_{n}\right| \leq c\left|g_{n}\right|$ for all $n \geq n_{0}$.
By Lemma 9,

$$
\begin{aligned}
& \operatorname{Pr}\left[\min _{i=1}^{I_{n}} Y_{i} \leq \mathcal{L}\left(\beta^{\prime}\right) \ln n\right] \\
& \lesssim \frac{1}{\sqrt{2 \pi \beta}} \frac{1}{\sqrt{\mu}(1-\mu)} \frac{I_{n}}{n \sqrt{\ln n}} n^{1-\left(\lambda_{n} / \ln n\right) \phi(\mu)} .
\end{aligned}
$$

Since

$$
1-\left(\lambda_{n} / \ln n\right) \phi(\mu) \rightarrow 1-\beta \phi(\mu)<0,
$$

we have

$$
\operatorname{Pr}\left[\min _{i=1}^{I_{n}} Y_{i} \leq \mathcal{L}\left(\beta^{\prime}\right) \ln n\right]=o(1) .
$$

Hence $\min _{i=1}^{I_{n}} Y_{i}>\mathcal{L}\left(\beta^{\prime}\right) \ln n$ a.a.s..

Next, assume that $I_{n}=O\left(\sqrt{\frac{n}{\ln n}}\right)$. Since $\mathcal{L}\left(2 \beta^{\prime}\right)<$ $\mathcal{L}(2 \beta)$, we have $\mathcal{L}\left(2 \beta^{\prime}\right) /(2 \beta)<\phi^{-1}(1 /(2 \beta))$. We choose a constant $\mu \in\left(\mathcal{L}\left(2 \beta^{\prime}\right) /(2 \beta), \phi^{-1}(1 /(2 \beta))\right)$. Thus, $\mu \in$ $(0,1), \mu \beta>\frac{1}{2} \mathcal{L}\left(2 \beta^{\prime}\right)$ and $\beta \phi(\mu)>1 / 2$. Thus, for sufficiently large $n, \mu \lambda_{n} \geq \frac{1}{2} \mathcal{L}\left(2 \beta^{\prime}\right) \ln n$, which implies that

$$
\begin{aligned}
\operatorname{Pr}\left[\min _{i=1}^{I_{n}} Y_{i} \leq \frac{1}{2} \mathcal{L}\left(2 \beta^{\prime}\right) \ln n\right] & \leq \operatorname{Pr}\left[\min _{i=1}^{I_{n}} Y_{i} \leq \mu \lambda_{n}\right] \\
& \leq I_{n} \operatorname{Pr}\left[Y \leq \mu \lambda_{n}\right] .
\end{aligned}
$$

By Lemma 9,

$$
\begin{aligned}
& \operatorname{Pr}\left[\min _{i=1}^{I_{n}} Y_{i} \leq \frac{1}{2} \mathcal{L}\left(2 \beta^{\prime}\right) \ln n\right] \\
& \lesssim \frac{1}{\sqrt{2 \pi \beta}} \frac{1}{\sqrt{\mu}(1-\mu)} \frac{I_{n}}{\sqrt{n \ln n}} n^{1 / 2-\left(\lambda_{n} / \ln n\right) \phi(\mu) .}
\end{aligned}
$$

Since

$$
1 / 2-\left(\lambda_{n} / \ln n\right) \phi(\mu) \rightarrow 1 / 2-\beta \phi(\mu)<0,
$$

we have

$$
\operatorname{Pr}\left[\min _{i=1}^{I_{n}} Y_{i} \leq \frac{1}{2} \mathcal{L}\left(2 \beta^{\prime}\right) \ln n\right]=o(1) .
$$

Hence $\min _{i=1}^{I_{n}} Y_{i}>\frac{1}{2} \mathcal{L}\left(2 \beta^{\prime}\right) \ln n$ a.a.s..

Finally, we consider that general case that $Y_{1}, Y_{2}, \cdots, Y_{I_{n}}$ have means $\lambda_{n, 1}, \lambda_{n, 2}, \cdots, \lambda_{n, I_{n}}$ respectively with $\lambda_{n, i} \geq \lambda_{n}$ for each $1 \leq i \leq I_{n}$. Let $Y_{1}^{\prime}, Y_{2}^{\prime}, \cdots, Y_{I_{n}}^{\prime}$ be $I_{n}$ Poisson RVs with means $\lambda_{n}$. For each $1 \leq i \leq I_{n}$, let $Y_{i}^{\prime \prime}$ be a Poisson RV with mean $\lambda_{n, i}-\lambda_{n}$ which is independent with $Y_{i}^{\prime}$. Then by the superposition property of Poisson RVs, $Y_{i}=$ $Y_{i}^{\prime}+Y_{i}^{\prime \prime}$. Therefore, $\min _{i=1}^{I_{n}} Y_{i} \geq \min _{i=1}^{I_{n}} Y_{i}^{\prime}>\mu \lambda_{n}$. By the above argument, the lemma also holds in this general case.

At the end of this subsection, we state the Palm theory [11] on the Poisson process.

Theorem 11: Let $n>0$. Suppose $k \in N$, and $h(\mathcal{Y}, \mathcal{X})$ is a bounded measurable function defined on all pairs of the form $(\mathcal{Y}, \mathcal{X})$ with $\mathcal{X} \subset \mathbb{R}^{2}$ being a finite subset and $\mathcal{Y}$ being a subset of $\mathcal{X}$, satisfying $h(\mathcal{Y}, \mathcal{X})=0$ except when $\mathcal{Y}$ has $k$ elements. Then

$$
\mathbf{E}\left[\sum_{\mathcal{Y} \subseteq \mathcal{P}_{n}} h\left(\mathcal{Y}, \mathcal{P}_{n}\right)\right]=\frac{n^{k}}{k !} \mathbf{E}\left[h\left(\mathcal{X}_{k}, \mathcal{X}_{k} \cup \mathcal{P}_{n}\right)\right]
$$

where the sum on the left-hand side is over all subsets $\mathcal{Y}$ of the random Poisson point set $\mathcal{P}_{n}$, and on the right hand side the set $\mathcal{X}_{k}$ is a binomial process with $k$ nodes, independent of $\mathcal{P}_{n}$. 
We need to estimate the number of subsets with some specified topology, for example, two nodes are local minima w.r.t. each other. But it is not so easy to estimate this among Poisson point processes. The Palm theory allows us to place a set of random points first and then estimate the expectation over the Poisson point process. This technique will be used in the proof of Lemma 4.

\section{Proof of Lemma 3}

To have the lower bound for minimal scan statistics, we apply the tessellation technique to discrete the scanning process. The deployment region is tessellated into equal-size square cells by properly choosing the cell size such that: (1) each copy of the lune contains a polyquadrates with area at least $c \frac{\ln n}{n}$ for some $c>1$ (or $\frac{1}{2} c \frac{\ln n}{n}$ if the copy crosses $\partial \mathbb{D}$ ), and (2) the number of polyquadrates is $O\left(\frac{n}{\ln n}\right)$ (or $O\left(\sqrt{\frac{n}{\ln n}}\right)$ if the copy crosses $\partial \mathbb{D})$. Then, the lemma follows Lemma 10. The detail is given below.

Proof: For a given $\beta_{1}$, choose a constant $\beta_{2} \in\left(\beta_{1}, \beta\right)$. Let $\varepsilon=\frac{1}{6 \sqrt{2} \beta_{0}}\left(1-\frac{\beta_{2}}{\beta}\right), d=\sqrt{3} r_{n}$, and consider an $\varepsilon d$ tessellation. (Note that $\varepsilon$ is chosen such that each copy of the lune contains a polyquadrate with area at least $c \ln n$ for some $c>1$.) Let $I_{n}$ denote the number of polyquadrates in $\mathbb{D}$ with span at most $\frac{1}{\varepsilon}$ and area at least $\frac{\beta_{2}}{\beta_{0}} \frac{\pi r_{n}^{2}}{\beta}=\left(\frac{\beta_{2}}{\beta_{0}}+o(1)\right) \frac{\ln n}{n}$, and $Y_{i}$ be the number of nodes on the $i$-th polyquadrate. Then $Y_{i}$ is a Poisson RV with rate at least $\left(\frac{\beta_{2}}{\beta_{0}}+o(1)\right) \ln n$. Since the number of cells in $\mathbb{D}$ is $O\left(\frac{n}{\ln n}\right)$, by Lemma 8 ,

$$
I_{n}=O\left(\left(\frac{1}{\varepsilon d}\right)^{2}\right)=O\left(\frac{n}{\ln n}\right) .
$$

By Lemma 10, it is a.a.s. that

$$
\frac{\min _{i=1}^{I_{n}} Y_{i}}{\ln n} \geq \mathcal{L}\left(\frac{\beta_{2}}{\beta_{0}}\right)>\mathcal{L}\left(\frac{\beta_{1}}{\beta_{0}}\right) .
$$

Now, let $I_{n}^{\prime}$ denote the number of polyquadrates in $\mathbb{D} \backslash \mathbb{D}_{-d}$ with span at most $\frac{1}{\varepsilon}$ and area at least $\frac{1}{2} \frac{\beta_{2}}{\beta_{0}} \frac{\pi r_{n}^{2}}{\beta}=$ $\frac{1}{2}\left(\frac{\beta_{2}}{\beta_{0}}+o(1)\right) \frac{\ln n}{n}$, and $Y_{i}^{\prime}$ be the number of nodes on the $i$-th polyquadrate. Then $Y_{i}^{\prime}$ is a Poisson RV with rate at least $\frac{1}{2}\left(\frac{\beta_{2}}{\beta_{0}}+o(1)\right) \ln n$. Since the number of cells in $\mathbb{D} \backslash \mathbb{D}_{-d}$ is $O\left(\sqrt{\frac{n}{\ln n}}\right)$, by Lemma 8 ,

$$
I_{n}^{\prime}=O\left(\frac{1}{\varepsilon d}\right)=O\left(\sqrt{\frac{n}{\ln n}}\right) .
$$

By Lemma 10, it is a.a.s. that

$$
\frac{\min _{i=1}^{I_{n}^{\prime}} Y_{i}^{\prime}}{\ln n} \geq \frac{1}{2} \mathcal{L}\left(\frac{\beta_{2}}{\beta_{0}}\right)>\frac{1}{2} \mathcal{L}\left(\frac{\beta_{1}}{\beta_{0}}\right) .
$$

Therefore, it is a.a.s. that

$$
\frac{\min \left(\min _{i=1}^{I_{n}} Y_{i}, \min _{i=1}^{I_{n}^{\prime}} Y_{i}^{\prime}\right)}{\ln n}>\frac{1}{2} \mathcal{L}\left(\frac{\beta_{1}}{\beta_{0}}\right) .
$$

Thus, the lemma follows if we can show that

$$
\mathcal{S}\left(\mathcal{P}_{n}, r_{n}\right) \geq \min \left(\min _{i=1}^{I_{n}} Y_{i}, \min _{i=1}^{I_{n}^{\prime}} Y_{i}^{\prime}\right) .
$$

To prove this inequality, it is sufficient to show that for any lune $L$ of two points in $\mathbb{D}$ which are separated by a distance of $r_{n}$, it either contains a polyquadrate in $\mathbb{D}$ with span at most $\frac{1}{\varepsilon}$ and area at least $\frac{\beta_{2}}{\beta_{0}} \frac{\pi r_{n}^{2}}{\beta}$, or contains a polyquadrate in $\mathbb{D} \backslash \mathbb{D}_{-d}$ with span at most $\frac{1}{\varepsilon}$ and area at least $\frac{1}{2} \frac{\beta_{2}}{\beta_{0}} \frac{\pi r_{n}^{2}}{\beta}$. We shall prove this in two cases.

Case 1: $L$ is contained in $\mathbb{D}$. Let $P$ denote the polyquadrate induced by $L_{-\sqrt{2} \varepsilon d}$. Then, $P \subseteq L \subseteq \mathbb{D}$, and the span of $P$ is at most $\left\lceil\frac{d-2 \sqrt{2} \varepsilon d}{\varepsilon d}\right\rceil+1 \leq \frac{1}{\varepsilon}$. By Lemma 7 and using the fact that $|L|=\pi r_{n}^{2} / \beta_{0}=\pi d^{2} /\left(3 \beta_{0}\right)$, we have

$$
\begin{aligned}
|P| & \geq\left|L_{-\sqrt{2} \varepsilon d}\right| \geq|L|-\pi d(\sqrt{2} \varepsilon d)=|L|-\sqrt{2} \varepsilon \pi d^{2} \\
& =|L|\left(1-3 \sqrt{2} \beta_{0} \varepsilon\right)>|L|\left(1-6 \sqrt{2} \beta_{0} \varepsilon\right)=\frac{\beta_{2}}{\beta}|L| \\
& =\frac{\beta_{2}}{\beta_{0}} \frac{\pi r_{n}^{2}}{\beta} .
\end{aligned}
$$

Case 2: $L$ is not contained in $\mathbb{D}$. Then $L$ must be disjoint with $\mathbb{D}_{-d}$. Let $L^{\prime}=L \cap \mathbb{D}$ and let $P^{\prime}$ denote the polyquadrate induced by $L_{-\sqrt{2} \varepsilon d}^{\prime}$. Then $P^{\prime} \subseteq L^{\prime} \subseteq \mathbb{D} \backslash \mathbb{D}_{-d}$ and the the span of $P$ is also at most $\frac{1}{\varepsilon}$. By Lemma 7 and Lemma 5, we have

$$
\begin{aligned}
\left|P^{\prime}\right| & \geq\left|L_{-\sqrt{2} \varepsilon d}^{\prime}\right| \geq\left|L^{\prime}\right|-\pi d(\sqrt{2} \varepsilon d) \geq \frac{1}{2}|L|-\sqrt{2} \pi \varepsilon d^{2} \\
& =\frac{1}{2}|L|\left(1-6 \sqrt{2} \beta_{0} \varepsilon\right)=\frac{1}{2} \frac{\beta_{2}}{\beta}|L|=\frac{1}{2} \frac{\beta_{2}}{\beta_{0}} \frac{\pi r_{n}^{2}}{\beta} .
\end{aligned}
$$

Thus, the lemma is proved.

\section{Proof of Lemma 4}

We introduce several relevant events and derive their probabilities. For convenience, we use $R_{1}$ and $R_{2}$ as shorthand for $R_{1}(n)$ and $R_{2}(n)$, respectively. Note that $\frac{1}{2} R_{2} \leq R_{1} \leq R_{2}$ and $\frac{\pi^{2}}{c^{2}}\left(1-\frac{R_{1}}{R_{2}}\right)<1$. Let $A$ denote the disk with radius $\frac{1}{2} \sqrt{\frac{\ln n}{n \pi}}$ at the center of the first cell. Assume $V$ is a point set and $T \subset V$. Let $h_{1}(T, V)$ denote a function such that $h_{1}\left(T=\left\{x_{1}, x_{2}\right\}, V\right)=1$ only if $\frac{1}{2}\left(x_{1}+x_{2}\right) \in A$, $R_{1} \leq\left\|x_{1}-x_{2}\right\| \leq R_{2}$, and there is no other node of $V$ in the lune area $L_{x_{1} x_{2}}$; otherwise, $h_{1}(T, V)=0$. Then, $E_{1}$ is the event that there exist two nodes $X, Y \in \mathcal{P}_{n}$ such that $h_{1}\left(\{X, Y\}, \mathcal{P}_{n}\right)=1$. In addition, under Boolean addition, for any $\left\{x_{1}, x_{2}, x_{3}\right\} \subseteq V$, let

$$
\begin{aligned}
h_{2}\left(\left\{x_{1}, x_{2}, x_{3}\right\}, V\right) & =h_{1}\left(\left\{x_{1}, x_{2}\right\}, V\right) \cdot h_{1}\left(\left\{x_{1}, x_{3}\right\}, V\right) \\
& +h_{1}\left(\left\{x_{2}, x_{1}\right\}, V\right) \cdot h_{1}\left(\left\{x_{2}, x_{3}\right\}, V\right) \\
& +h_{1}\left(\left\{x_{3}, x_{1}\right\}, V\right) \cdot h_{1}\left(\left\{x_{3}, x_{2}\right\}, V\right) ;
\end{aligned}
$$

for any $\left\{x_{1}, x_{2}, x_{3}, x_{4}\right\} \subseteq V$, let

$$
\begin{aligned}
h_{3}\left(\left\{x_{1}, x_{2}, x_{3}, x_{4}\right\}, V\right) & =h_{1}\left(\left\{x_{1}, x_{2}\right\}, V\right) \cdot h_{1}\left(\left\{x_{3}, x_{4}\right\}, V\right) \\
& +h_{1}\left(\left\{x_{1}, x_{3}\right\}, V\right) \cdot h_{1}\left(\left\{x_{2}, x_{4}\right\}, V\right) \\
& +h_{1}\left(\left\{x_{1}, x_{4}\right\}, V\right) \cdot h_{1}\left(\left\{x_{2}, x_{3}\right\}, V\right) .
\end{aligned}
$$

For the sake of clarity, in the remaining of this subsection, we use $X_{1}, X_{2}, X_{3}$ and $X_{4}$ to denote independent random points with uniform distribution over $\mathbb{D}$ and independent of $\mathcal{P}_{n}$, and $X_{1}^{\prime}, X_{2}^{\prime}, X_{3}^{\prime}$ and $X_{4}^{\prime}$ to denote elements of $\mathcal{P}_{n}$. Let $F_{1}^{\prime}\left(\left\{X_{1}^{\prime}, X_{2}^{\prime}\right\}\right)$ be the event that $h_{1}\left(\left\{X_{1}^{\prime}, X_{2}^{\prime}\right\}, \mathcal{P}_{n}\right)=1 ; F_{2}^{\prime}\left(\left\{X_{1}^{\prime}, X_{2}^{\prime}, X_{3}^{\prime}\right\}\right)$ be the event 
that $h_{2}\left(\left\{X_{1}^{\prime}, X_{2}^{\prime}, X_{3}^{\prime}\right\}, \mathcal{P}_{n}\right)=1$; and $F_{3}^{\prime}\left(\left\{X_{1}^{\prime}, X_{2}^{\prime}, X_{3}^{\prime}, X_{4}^{\prime}\right\}\right)$ be the event that $h_{3}\left(\left\{X_{1}^{\prime}, X_{2}^{\prime}, X_{3}^{\prime}, X_{4}^{\prime}\right\}, \mathcal{P}_{n}\right)=1$. Applying Boole's inequalities which is a special case of the inclusionexclusion principle, we have

$$
\begin{aligned}
\operatorname{Pr}\left[E_{1}\right] \geq & \sum_{\left\{X_{1}^{\prime}, X_{2}^{\prime}\right\} \subseteq \mathcal{P}_{n}} \operatorname{Pr}\left[F_{1}^{\prime}\left(\left\{X_{1}^{\prime}, X_{2}^{\prime}\right\}\right)\right] \\
& -\sum_{\left\{X_{1}^{\prime}, X_{2}^{\prime}, X_{3}^{\prime}\right\} \subseteq \mathcal{P}_{n}} \operatorname{Pr}\left[F_{2}^{\prime}\left(\left\{X_{1}^{\prime}, X_{2}^{\prime}, X_{3}^{\prime}\right\}\right)\right] \\
& -\sum_{\left\{X_{1}^{\prime}, X_{2}^{\prime}, X_{3}^{\prime}, X_{4}^{\prime}\right\} \subseteq \mathcal{P}_{n}} \operatorname{Pr}\left[F_{3}^{\prime}\left(\left\{X_{1}^{\prime}, X_{2}^{\prime}, X_{3}^{\prime}, X_{4}^{\prime}\right\}\right)\right] .
\end{aligned}
$$

Let $F_{1}$ be the event that $h_{1}\left(\left\{X_{1}, X_{2}\right\},\left\{X_{1}, X_{2}\right\} \cup \mathcal{P}_{n}\right)=1, F_{2}$ be the event that $h_{2}\left(\left\{X_{1}, X_{2}, X_{3}\right\},\left\{X_{1}, X_{2}, X_{3}\right\} \cup \mathcal{P}_{n}\right)=1$, and $F_{3}$ be the event that $h_{3}\left(\left\{X_{1}, X_{2}, X_{3}, X_{4}\right\},\left\{X_{1}, X_{2}, X_{3}, X_{4}\right\} \cup \mathcal{P}_{n}\right)=$ 1. According to the Palm theory (Theorem 11), we have

$$
\begin{aligned}
& \sum_{\left\{X_{1}^{\prime}, X_{2}^{\prime}\right\} \subseteq \mathcal{P}_{n}} \operatorname{Pr}\left[F_{1}^{\prime}\left(\left\{X_{1}^{\prime}, X_{2}^{\prime}\right\}\right)\right] \\
= & \mathbf{E}\left[\sum_{\left\{X_{1}^{\prime}, X_{2}^{\prime}\right\} \subseteq \mathcal{P}_{n}} h_{1}\left(\left\{X_{1}^{\prime}, X_{2}^{\prime}\right\}, \mathcal{P}_{n}\right)\right] \\
= & \frac{n^{2}}{2 !} \mathbf{E}\left[h_{1}\left(\left\{X_{1}, X_{2}\right\},\left\{X_{1}, X_{2}\right\} \cup \mathcal{P}_{n}\right)\right] \\
= & \frac{n^{2}}{2} \operatorname{Pr}\left[F_{1}\right] ;
\end{aligned}
$$

$$
\begin{aligned}
& \sum_{\left\{X_{1}^{\prime}, X_{2}^{\prime}, X_{3}^{\prime}\right\} \subseteq \mathcal{P}_{n}} \operatorname{Pr}\left[F_{2}^{\prime}\left(\left\{X_{1}^{\prime}, X_{2}^{\prime}, X_{3}^{\prime}\right\}\right)\right] \\
& =\mathbf{E}\left[\sum_{\left\{X_{1}^{\prime}, X_{2}^{\prime}, X_{3}^{\prime}\right\} \subseteq \mathcal{P}_{n}} h_{2}\left(\left\{X_{1}^{\prime}, X_{2}^{\prime}, X_{3}^{\prime}\right\}, \mathcal{P}_{n}\right)\right] \\
& =\frac{n^{3}}{3 !} \mathbf{E}\left[h_{2}\left(\left\{X_{1}, X_{2}, X_{3}\right\},\left\{X_{1}, X_{2}, X_{3}\right\} \cup \mathcal{P}_{n}\right)\right] \\
& =3 \frac{n^{3}}{3 !} \operatorname{Pr}\left[F_{2}\right]=\frac{n^{3}}{2} \operatorname{Pr}\left[F_{2}\right] \text {; }
\end{aligned}
$$

and

$$
\begin{aligned}
& \sum_{\left\{X_{1}^{\prime}, X_{2}^{\prime}, X_{3}^{\prime}, X_{4}^{\prime}\right\} \subseteq \mathcal{P}_{n}} \operatorname{Pr}\left[F_{3}^{\prime}\left(\left\{X_{1}^{\prime}, X_{2}^{\prime}, X_{3}^{\prime}, X_{4}^{\prime}\right\}\right)\right] \\
& =\mathbf{E}\left[\sum_{\left\{X_{1}^{\prime}, X_{2}^{\prime}, X_{3}^{\prime}, X_{4}^{\prime}\right\} \subseteq \mathcal{P}_{n}} h_{3}\left(\left\{X_{1}^{\prime}, X_{2}^{\prime}, X_{3}^{\prime}, X_{4}^{\prime}\right\}, \mathcal{P}_{n}\right)\right] \\
& =\frac{n^{4}}{4 !} \mathbf{E}\left[h_{3}\left(\left\{X_{1}, X_{2}, X_{3}, X_{4}\right\},\left\{X_{1}, X_{2}, X_{3}, X_{4}\right\} \cup \mathcal{P}_{n}\right)\right] \\
& =3 \frac{n^{4}}{4 !} \operatorname{Pr}\left[F_{3}\right]=\frac{n^{4}}{8} \operatorname{Pr}\left[F_{3}\right] \text {. }
\end{aligned}
$$

From Eq. (6), (7), (8), and (9), we have

$$
\operatorname{Pr}\left[E_{1}\right] \geq \frac{n^{2}}{2} \operatorname{Pr}\left[F_{1}\right]-\frac{n^{3}}{2} \operatorname{Pr}\left[F_{2}\right]-\frac{n^{4}}{8} \operatorname{Pr}\left[F_{3}\right] .
$$

In the next, we derive the probabilities of $F_{1}, F_{2}$, and $F_{3}$. Let $S_{1}\left(R_{1}, R_{2}\right)$ denote the set

$$
\left\{\left(x_{1}, x_{2}\right) \mid \frac{1}{2}\left(x_{1}+x_{2}\right) \in A, R_{1} \leq\left\|x_{1}-x_{2}\right\| \leq R_{2}\right\} .
$$

For simplicity, $S_{1}$ is shorthand for $S_{1}\left(R_{1}, R_{2}\right)$. We have

$$
\begin{aligned}
\operatorname{Pr}\left[F_{1}\right] & =\iint_{S_{1}} \operatorname{Pr}\left[F_{1} \mid X_{1}=x_{1}, X_{2}=x_{2}\right] d x_{1} d x_{2} \\
& =\iint_{S_{1}} e^{-n\left|L_{x_{1} x_{2}}\right|} d x_{1} d x_{2} \\
& =\iint_{S_{1}} e^{-n \frac{1}{\beta_{0}} \pi\left\|x_{1}-x_{2}\right\|^{2}} d x_{1} d x_{2} .
\end{aligned}
$$

Let $z=\frac{x_{1}+x_{2}}{2}$ and $r=\frac{1}{2}\left\|x_{1}-x_{2}\right\|$. Then,

$$
\begin{aligned}
\operatorname{Pr}\left[F_{1}\right] & =\int_{z \in A} \int_{r=\frac{R_{1}}{2}}^{\frac{R_{2}}{2}} e^{-\frac{4}{\beta_{0}} n \pi r^{2}} 8 \pi r d r d z \\
& =4 \int_{z \in A} \int_{r=\frac{R_{1}}{2}}^{\frac{R_{2}}{2}} e^{-\frac{4}{\beta_{0}} n \pi r^{2}} 2 \pi r d r d z \\
& =4 \int_{z \in A} \int_{r=\frac{R_{1}}{2}}^{\frac{R_{2}}{2}} e^{-\frac{4}{\beta_{0}} n \pi r^{2}} d\left(\pi r^{2}\right) d z \\
& =-\left(\left.\frac{\beta_{0}}{n} e^{-\frac{4}{\beta_{0}} n \pi r^{2}}\right|_{r=\frac{R_{1}}{2}} ^{\frac{R_{2}}{2}}\right)|A| \\
& =\frac{\beta_{0}}{4 n^{2}}\left(n^{-\frac{\beta_{1}}{\beta_{0}}}-n^{-\frac{\beta_{2}}{\beta_{0}}}\right) \ln n .
\end{aligned}
$$

Let $S_{2}\left(R_{1}, R_{2}\right)$ denote the set

$$
\left\{\begin{array}{c|c}
\left(x_{1}, x_{2}, x_{3}\right) & \begin{array}{c}
\frac{x_{1}+x_{2}}{2}, \frac{x_{1}+x_{3}}{2} \in A ; \\
R_{1} \leq\left\|x_{1}-x_{2}\right\| \leq R_{2} ; \\
R_{1} \leq\left\|x_{1}-x_{3}\right\| \leq R_{2} ; x_{1}, x_{2} \\
x_{1}, x_{3} \notin L_{x_{1} x_{3}} ;
\end{array}
\end{array}\right\} .
$$

Again, for simplicity, $S_{2}$ is shorthand for $S_{2}\left(R_{1}, R_{2}\right)$. Applying Lemma 6, if $\left(x_{1}, x_{2}, x_{3}\right) \in S_{2}$, we have

$$
\begin{aligned}
& \operatorname{Pr}\left[F_{2} \mid X_{1}=x_{1}, X_{2}=x_{2}, X_{3}=x_{3}\right] \leq e^{-n\left|L_{x_{1} x_{2}} \cup L_{x_{1} x_{3}}\right|} \\
& \leq e^{-n\left(\frac{1}{\beta_{0}} \pi\left\|x_{1}-x_{2}\right\|^{2}+c R_{2}\left\|\frac{x_{1}+x_{2}}{2}-\frac{x_{1}+x_{3}}{2}\right\|\right)} .
\end{aligned}
$$

Therefore,

$$
\begin{aligned}
& \operatorname{Pr}\left[F_{2}\right] \\
& =\iiint_{S_{2}} \operatorname{Pr}\left[F_{2} \mid X_{1}=x_{1}, X_{2}=x_{2}, X_{3}=x_{3}\right] d x_{1} d x_{2} d x_{3} \\
& \leq \iiint_{S_{2}} e^{-n\left(\frac{1}{\beta_{0}} \pi\left\|x_{1}-x_{2}\right\|^{2}+c R_{2}\left\|\frac{x_{1}+x_{2}}{2}-\frac{x_{1}+x_{3}}{2}\right\|\right)} d x_{1} d x_{2} d x_{3} .
\end{aligned}
$$

Let $z_{1}=\frac{x_{1}+x_{2}}{2}, r_{1}=\frac{1}{2}\left\|x_{1}-x_{2}\right\|, z_{2}=\frac{x_{1}+x_{3}}{2}$, and $\rho=$ $\left\|z_{1}-z_{2}\right\|$. Then,

$$
\begin{aligned}
& \operatorname{Pr}\left[F_{2}\right] \\
& \leq 16 \int_{z_{1} \in A} \int_{r_{1}=\frac{R_{1}}{2}}^{\frac{R_{2}}{2}} \int_{z_{2} \in A} e^{-n\left(\frac{4}{\beta_{0}} \pi r_{1}^{2}+c R_{2}\left\|z_{1}-z_{2}\right\|\right)} 2 \pi r_{1} \\
& \quad \cdot d r_{1} d z_{1} d z_{2} \\
& \leq 16 \int_{z_{1} \in A} \int_{r_{1}=\frac{R_{1}}{2}}^{\frac{R_{2}}{2}} e^{-\frac{4}{\beta_{0}} n \pi r_{1}^{2}} 2 \pi r_{1} d r_{1} d z_{1} \\
& \quad \cdot \int_{z_{2} \in A} e^{-c n R_{2}\left\|z_{1}-z_{2}\right\|} d z_{2} \\
& \leq 16 \int_{z_{1} \in A} \int_{r_{1}=\frac{R_{1}}{2}}^{\frac{R_{2}}{2}} e^{-\frac{4}{\beta_{0}} n \pi r_{1}^{2}} d\left(\pi r_{1}^{2}\right) d z_{1}
\end{aligned}
$$




$$
\begin{aligned}
& \int_{\rho=0}^{\infty} e^{-c n R_{2} \rho} 2 \pi \rho d \rho \\
= & -\left(\left.\frac{4 \beta_{0}}{n} e^{-\frac{4}{\beta_{0}} n \pi r^{2}}\right|_{r=\frac{R_{1}}{2}} ^{\frac{R_{2}}{2}}|A| \frac{2 \pi}{\left(c n R_{2}\right)^{2}}\right. \\
= & \frac{2 \pi \beta_{0}}{c^{2}\left(n R_{2}^{2}\right) n^{3}}\left(n^{-\frac{\beta_{1}}{\beta_{0}}}-n^{-\frac{\beta_{2}}{\beta_{0}}}\right) \ln n .
\end{aligned}
$$

Let $S_{3}\left(R_{1}, R_{2}\right)$ denote the set

$$
\left\{\begin{array}{c|c}
\left(x_{1}, x_{2}, x_{3}, x_{4}\right) & \frac{x_{1}+x_{2}}{2}, \frac{x_{3}+x_{4}}{2} \in A ; \\
R_{1} \leq\left\|x_{1}-x_{2}\right\| \leq R_{2} ; \\
R_{1} \leq\left\|x_{3}-x_{4}\right\| \leq R_{2} ; \\
x_{1}, x_{2} \notin L_{x_{3} x_{4}} ; x_{3}, x_{4} \notin L_{x_{1} x_{2}}
\end{array}\right\} .
$$

Again, for simplicity, $S_{3}$ is shorthand for $S_{3}\left(R_{1}, R_{2}\right)$. Applying Lemma 6 , if $\left(x_{1}, x_{2}, x_{3}, x_{4}\right) \in S_{3}$, we have

$$
\begin{aligned}
& \operatorname{Pr}\left[F_{3} \mid X_{1}=x_{1}, X_{2}=x_{2}, X_{3}=x_{3}, X_{4}=x_{4}\right] \\
& \leq e^{-n\left|L_{x_{1} x_{2}} \cup L_{x_{3} x_{4}}\right|} \\
& \leq e^{-n\left(\frac{1}{\beta_{0}} \pi\left\|x_{1}-x_{2}\right\|^{2}+c R_{2}\left\|\frac{x_{1}+x_{2}}{2}-\frac{x_{3}+x_{4}}{2}\right\|\right)} .
\end{aligned}
$$

Therefore,

$$
\begin{aligned}
& \operatorname{Pr}\left[F_{3}\right] \\
& =\iiint \int_{S_{3}} \operatorname{Pr}\left[F_{3} \mid X_{1}=x_{1}, X_{2}=x_{2}, X_{3}=x_{3}, X_{4}=x_{4}\right] \\
& \quad \cdot d x_{1} d x_{2} d x_{3} d x_{4} \\
& \leq \iiint \int_{S_{3}} e^{-n\left(\frac{1}{\beta_{0}} \pi\left\|x_{1}-x_{2}\right\|^{2}+c R_{2}\left\|\frac{x_{1}+x_{2}}{2}-\frac{x_{3}+x_{4}}{2}\right\|\right)} \\
& \quad \cdot d x_{1} d x_{2} d x_{3} d x_{4} .
\end{aligned}
$$

Let $z_{1}=\frac{x_{1}+x_{2}}{2}, r_{1}=\frac{1}{2}\left\|x_{1}-x_{2}\right\|, z_{2}=\frac{x_{3}+x_{4}}{2}, r_{2}=$ $\frac{1}{2}\left\|x_{3}-x_{4}\right\|$, and $\rho=\left\|z_{1}-z_{2}\right\|$. Then,

$$
\begin{aligned}
\operatorname{Pr} & {\left[F_{3}\right] } \\
\leq & \int_{z_{1} \in A} \int_{r_{1}=\frac{R_{1}}{2}}^{\frac{R_{2}}{2}} \int_{z_{2} \in A} \int_{r_{2}=\frac{R_{1}}{2}}^{\frac{R_{2}}{2}} e^{-n\left(\frac{4}{\beta_{0}} \pi r_{1}^{2}+c R_{2}\left\|z_{1}-z_{2}\right\|\right)} \\
& \cdot\left(8 \pi r_{1} d r_{1} d z_{1}\right)\left(8 \pi r_{2} d r_{2} d z_{2}\right) \\
\leq & \left(4 \int_{z_{1} \in A} \int_{r_{1}=\frac{R_{1}}{2}}^{\frac{R_{2}}{2}} e^{-\frac{4}{\beta_{0}} n \pi r_{1}^{2}} 2 \pi d r_{1} d z_{1}\right) \\
& \cdot\left(8 \pi \frac{R_{2}}{2}\left(\frac{R_{2}}{2}-\frac{R_{1}}{2}\right) \int_{z_{2} \in A} e^{-c n R_{2}\left\|z_{1}-z_{2}\right\|} d z_{2}\right) \\
\leq & \left(4 \int_{z_{1} \in A} \int_{r_{1}=\frac{R_{1}}{2}}^{\frac{R_{2}}{2}} e^{-\frac{4}{\beta_{0}} n \pi r_{1}^{2}} d\left(\pi r_{1}^{2}\right) d z_{1}\right) \\
& \cdot\left(8 \pi \frac{R_{2}}{2}\left(\frac{R_{2}}{2}-\frac{R_{1}}{2}\right) \int_{\rho=0}^{\infty} e^{-c n R_{2} \rho} 2 \pi \rho d \rho\right) \\
= & \left(\frac{\beta_{0} \ln n}{4 n^{2}}\left(n^{-\frac{\beta_{1}}{\beta_{0}}}-n^{-\frac{\beta_{2}}{\beta_{0}}}\right)\right)\left(\frac{4 \pi^{2}}{\left(c n R_{2}\right)^{2}} R_{2}\left(R_{2}-R_{1}\right)\right) \\
= & \frac{\pi^{2} \beta_{0}}{c^{2} n^{4}}\left(1-\frac{R_{1}}{R_{2}}\right)\left(n^{-\frac{\beta_{1}}{\beta_{0}}}-n^{-\frac{\beta_{2}}{\beta_{0}}}\right) \ln n .
\end{aligned}
$$

Put Eq. (10), (11), (12) and (13) together. We have

$$
\begin{aligned}
& \operatorname{Pr}\left[E_{1}\right] \\
& \geq\left(\frac{\beta_{0}}{8}-\frac{\pi \beta_{0}}{c^{2}\left(n R_{2}^{2}\right)}-\frac{\pi^{2} \beta_{0}}{8 c^{2}}\left(1-\frac{R_{1}}{R_{2}}\right)\right)\left(n^{-\frac{\beta_{1}}{\beta_{0}}}-n^{-\frac{\beta_{2}}{\beta_{0}}}\right) \ln n \\
& \sim \frac{\beta_{0}}{8}\left(1-\frac{\pi^{2}}{c^{2}}\left(1-\frac{R_{1}}{R_{2}}\right)\right)\left(n^{-\frac{\beta_{1}}{\beta_{0}}}-n^{-\frac{\beta_{2}}{\beta_{0}}}\right) \ln n .
\end{aligned}
$$

Recall that for a given $\beta, \beta_{1}$ and $\beta_{2}$ are constants, and so are $\frac{\beta_{1}}{\beta_{0}}$ and $\frac{\beta_{2}}{\beta_{0}}$. According to Eq.(3), $\frac{R_{1}}{R_{2}}$ also is a constant. We write $f_{n}=\Omega\left(g_{n}\right)$ for two sequences $f_{n}$ and $g_{n}$ if there exist constant $c_{1}>0$ and $n_{0}$ such that $\left|f_{n}\right| \geq c_{1}\left|g_{n}\right|$ for all $n \geq n_{0}$. From Eq. (2), we have

$$
\operatorname{Pr}\left[E_{1}\right]=\Omega\left(\left(n^{-\frac{\beta_{1}}{\beta_{0}}}-n^{-\frac{\beta_{2}}{\beta_{0}}}\right) \ln n\right) .
$$

Since $I_{n}=\Omega\left(\frac{n}{\ln n}\right)$ from Eq. (4), we have

$$
I_{n} \operatorname{Pr}\left[E_{1}\right]=\Omega\left(n^{1-\frac{\beta_{1}}{\beta_{0}}}\right) \rightarrow \infty .
$$

This complete the proof of Lemma 4.

\section{ACKNOWLEDGMENT}

The work of P.-J. Wan is supported in part by the NSF under Grant 557904 and CityU of Hong Kong under Grant 7200031 .

This work of C.-W. Yi is supported in part by the NSC under Grant No. NSC95-2221-E-009-059-MY3 and NSC97-2221-E009-052-MY3, by the ITRI under Grant No. 7352B12100, and by the MoE ATU plan.

The work of F. Yao described in this paper was partially supported by grants from the Research Grants Council of the Hong Kong SAR, China, under Project No. 122105, 122807, and the National Basic Research Program of China Grant 2007CB807900, 2007CB807901.

The work of X. Jia is partially supported by a grant from Research Grants Council of Hong Kong [Project No. CityU 114006].

\section{REFERENCES}

[1] H. Takagi and L. Kleinrock, "Optimal transmission ranges for randomly distributed packet radio terminals," IEEE Trans. Commun., vol. COM32, no. 3, pp. 246-257, Mar. 1984.

[2] G. Xing, C. Lu, R. Pless, and Q. Huang, "On greedy geographic routing algorithms in sensing-covered networks," in Proc. 5th ACM International Symp. Mobile Ad Hoc Networking Computing, May 2004, pp. 31-42.

[3] M. de Berg, M. van Kreveld, M. Overmars, and O. Schwarzkopf, Computational Geometry: Algorithms and Applications. Springer-Verlag, Mar. 2008.

[4] G. Finn, "Routing and addressing problems in large metropolitan-scale internetworks," University of Southern California, Marina del Rey. Information Sciences Institute, Tech. Rep. ISI/RR-87-180, Mar. 1987.

[5] B. Karp and H. Kung, "GPSR: greedy perimeter stateless routing for wireless networks," in Proc. 6th Annual International Conf. Mobile Computing Networking (MobiCom 2000), pp. 243-254.

[6] M. D. Penrose, "The longest edge of the random minimal spanning tree," Annals Applied Probability, vol. 7, no. 2, pp. 340-361, May 1997.

[7] _ _, "A strong law for the longest edge of the minimal spanning tree," Annals Probability, vol. 27, no. 1, pp. 246-260, Jan. 1999.

[8] P. Gupta and P. R. Kumar, "Critical power for asymptotic connectivity in wireless networks," Stochastic Analysis, Control, Optimization and Applications: A Volume in Honor of W. H. Fleming, W. M. McEneaney, G. Yin, and Q. Zhang, eds. Birkhauser, Mar. 1998, pp. 547-566.

[9] F. Baccelli and C. Bordenave, "The radial spanning tree of a poisson point process," Annals Applied Probability, vol. 17, no. 1, pp. 305-359, Feb. 2007.

[10] P.-J. Wan, C.-W. Yi, F. Yao, and X. Jia, "Asymptotic critical transmission radius for greedy forward routing in wireless ad hoc networks," in Proc. 7th ACM International Symp. Mobile Ad Hoc Networking Computing, May 2006, pp. 25-36.

[11] F. Baccelli and P. Bremaud, Elements of Queueing Theory: PalmMartingale Calculus and Stochastic Recurrences. Springer, 2003. 

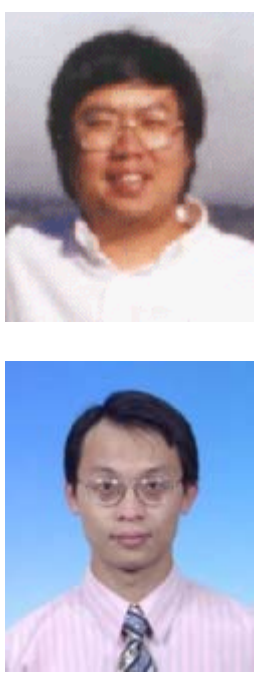

Peng-Jun Wan received his PhD degree from University of Minnesota, MS degree from The Chinese Academy of Science, and BS degree from Tsinghua University. He is currently an Associate Professor in Computer Science at Illinois Institute of Technology, and at City University of Hong Kong. His research interests include wireless networks, optical networks, and algorithm design and analysis.

Chih-Wei Yi received his Ph.D. degree from the Illinois Institute of Technology, and MS and BS degrees from the National Taiwan University. He is currently an Assistant Professor in Computer Science at the National Chiao Tung University. His research focuses on wireless ad hoc and sensor networks.

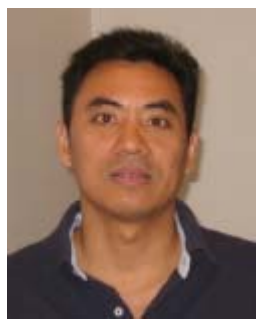

Lixin Wang received the M.S. degree in CS from the University of Houston at Clear Lake, the M.S. degree in Applied Math from the University of Houston and the M.S. degree in Math from the Fudan University, Shanghai, China. He is currently a Ph.D. student in Computer Science at the Illinois Institute of Technology, Chicago. His research is on wireless networks, and algorithm design and analysis.

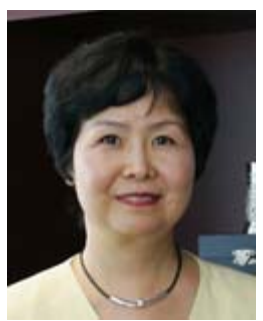

Frances Yao received her BSc (1969) from National Taiwan University and her Ph.D. (1973) in Mathematics from Massachusetts Institute of Technology. She is currently Head of the Department of Computer Science at City University of Hong Kong. Her research interests include combinatorial and geometric algorithms, energy-efficient computing and sensor networks. She is a Fellow of AAAS.

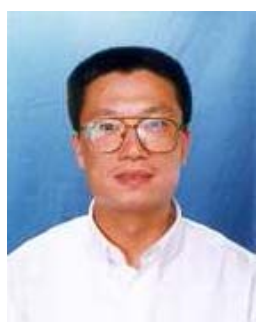

Xiaohua Jia received his BSc (1984) and MEng (1987) from the Univ of Science and Technolog of China, and obtained his DSc (1991) in Information Science from the Univ. of Tokyo, Japan. Prof. Jia is currently associated with Dept of Computer Science at City Univ of Hong Kong. His research interests include distributed systems, computer networks, WDM optical networks, and Internet and mobile computing. 